\title{
Superconductivity-induced transverse plasma mode and phonon anomaly in the $c$-axis response of the bilayer compound $\mathrm{RbCa}_{2} \mathrm{Fe}_{4} \mathrm{As}_{4} \mathrm{~F}_{2}$
}

\author{
B. Xu, ${ }^{1}$ D. Munzar, ${ }^{2}$ A. Dubroka $\odot,{ }^{2}$ E. Sheveleva $\odot,{ }^{1}$ F. Lyzwa,,${ }^{1}$ P. Marsik, ${ }^{1}$ C. N. Wang, ${ }^{3}$ Z. C. Wang, ${ }^{4}$ \\ G. H. Cao, ${ }^{4}$ and C. Bernhard ${ }^{1, *}$ \\ ${ }^{1}$ Department of Physics and Fribourg Center for Nanomaterials, University of Fribourg, Chemin du Musée 3, 1700 Fribourg, Switzerland \\ ${ }^{2}$ Department of Condensed Matter Physics, Faculty of Science, and Central European Institute of Technology, Masaryk University, \\ Kotlářská 2, 61137 Brno, Czech Republic \\ ${ }^{3}$ Laboratory for Muon Spin Spectroscopy, Paul Scherrer Institute, 5232 Villigen PSI, Switzerland \\ ${ }^{4}$ Department of Physics and State Key Lab of Silicon Materials, Zhejiang University, Hangzhou 310027, China
}

(Received 18 April 2020; accepted 28 May 2020; published 9 June 2020)

\begin{abstract}
We studied the infrared response of polycrystalline samples of the iron arsenide superconductor ( $\mathrm{Rb}, \mathrm{Cs}) \mathrm{Ca}_{2} \mathrm{Fe}_{4} \mathrm{As}_{4} \mathrm{~F}_{2}$ (Rb,Cs-12442), which has a bilayer structure similar to the high- $T_{c}$ cuprates $\mathrm{YBa}_{2} \mathrm{Cu}_{3} \mathrm{O}_{7}$ (YBCO) and $\mathrm{Bi}_{2} \mathrm{Sr}_{2} \mathrm{CaCu}_{2} \mathrm{O}_{8}$. The $c$-axis reflectivity spectra $R_{c}$ have been derived from the reflectivity spectra of the polycrystalline samples $R_{\text {poly }}$ and the in-plane spectrum of a corresponding Cs- 12442 crystal $R_{a b}$ using a geometrical averaging approach with $R_{c}=3 R_{\text {poly }}-2 R_{a b}$. In analogy to the $c$-axis response of the cuprates, we observe a superconductivity-induced transverse plasma mode and a phonon anomaly that are both signatures of local electric field effects that arise from a large difference between the local conductivities in the intraand interbilayer regions. Using a multilayer model developed for the cuprates, we obtain a good description of the $c$-axis response and derive the local conductivities at $T \simeq T_{c}$ of $\sigma_{1}^{\mathrm{bl}}(\omega \rightarrow 0) \simeq 1000 \Omega^{-1} \mathrm{~cm}^{-1}$ and $\sigma_{1}^{\text {int }}(\omega \rightarrow 0) \simeq 15 \Omega^{-1} \mathrm{~cm}^{-1}$, respectively, that are similar to the ones previously found in underdoped YBCO. Different from the cuprates, we find no evidence of a normal-state pseudogap in terms of a partial suppression of the low-energy electronic states that sets in already well above $T_{c}$. There is also no clear sign of an onset of precursor superconducting pairing correlations well above $T_{c} \simeq 30 \mathrm{~K}$. This highlights that the pseudogap and the precursor superconducting pairing well above $T_{c}$ are unique features of the cuprates with their strong electronic correlations and, for example, not just the result of a strongly anisotropic electronic response due to the layered crystal structure.
\end{abstract}

DOI: 10.1103/PhysRevB.101.214512

\section{INTRODUCTION}

\section{A. $c$-axis response of cuprates}

The study of the infrared $c$-axis response of the cuprate high- $T_{c}$ superconductors, like $\mathrm{La}_{2-x} \mathrm{Sr}_{x} \mathrm{CuO}_{4}$ (LSCO) and $\mathrm{YBa}_{2} \mathrm{Cu}_{3} \mathrm{O}_{7-\delta}$ (YBCO), has provided valuable information about their unusual electronic properties in the normal and superconducting states. The incoherent, insulatorlike response in the normal state and the emergence of a rather sharp plasma edge in the low-frequency $c$-axis reflectivity below $T_{c}$ are, meanwhile, understood in terms of a weak, Josephson-type coupling between the quasi-two-dimensional $\mathrm{CuO}_{2}$ planes. Both the incoherence of the $c$-axis response in the normal state and the very small value of the longitudinal superconducting (SC) plasma frequency below $T_{c}$ are due to the so-called pseudogap phenomenon, which gives rise to a partial depletion of the low-energy electronic states that sets in far above $T_{c}$ and persists in the SC state [1-10].

The origin of this pseudogap, which governs the unusual electronic properties in the so-called underdoped part of the phase diagram, has been investigated for decades and remains

\footnotetext{
*christian.bernhard@unifr.ch
}

debated. Meanwhile, at least some phenomenological trends are commonly accepted. The pseudogap affects only parts of the Fermi surface of the cuprates; that is, it is largest in the so-called antinodal region close to $(0, \pi)$ or $(\pi, 0)$ and absent in the nodal region close to the Brillouin-zone diagonal (in the notation of the $d$-wave superconducting order parameter) [11]. The pseudogap has, therefore, a strong influence on the $c$-axis conductivity, especially in YBCO, for which the matrix element for the hopping across the spacing layer including the $\mathrm{BaO}$ and $\mathrm{CuO}$ chain layers displays a similar $k$-space anisotropy; that is, it is large (small) in the antinodal (nodal) regions of the Fermi surface. Notably, as the pseudogap vanishes on the overdoped side of the phase diagram, the normal-state $c$-axis conductivity becomes metal-like, albeit with a much smaller (longitudinal) SC plasma frequency than for the in-plane response.

Several additional, striking features have been observed in the $c$-axis response of the so-called bilayer compounds, like YBCO and $\mathrm{Bi}_{2} \mathrm{Sr}_{2} \mathrm{CaCu}_{2} \mathrm{O}_{8}$ (Bi-2212) [7-10,12,13]. They contain pairs of closely spaced $\mathrm{CuO}_{2}$ layers (so-called bilayers) that are separated by an $\mathrm{Y}$ or $\mathrm{Ca}$ monolayer, respectively. The electronic coupling within the $\mathrm{CuO}_{2}$ bilayer unit is thus considerably stronger than the one across the thicker stacks of $\mathrm{BaO}_{2}-\mathrm{CuO}-\mathrm{BaO}_{2}$ layers in $\mathrm{YBCO}$ and $\mathrm{SrO}_{2}-\mathrm{BiO}_{2}-\mathrm{SrO}_{2}$ 
layers in $\mathrm{Bi}-2212$ that separate the $\mathrm{CuO}_{2}$ bilayer units and are denoted as interbilayer regions in the following. This leads to large differences between the local conductivities in the intrabilayer and interbilayer regions, $\sigma_{1}^{\text {bl }}$ and $\sigma_{1}^{\text {int }}$, and the related plasma frequencies, $\omega_{p}^{\mathrm{bl}}$ and $\omega_{p}^{\text {int }}$, respectively. Accordingly, there exists a transverse plasma mode (tPM) that shows up as a peak in the optical conductivity at a frequency $\omega_{p}^{\mathrm{tr}}$, with $\omega_{p}^{\mathrm{bl}}>\omega_{p}^{\mathrm{tr}}>\omega_{p}^{\mathrm{int}}$, at which the currents in the intraand interbilayer regions oscillate out of phase (the interbilayer response may also be insulating with $\omega_{p}^{\text {int }}=0$ ) [14]. In the incoherent normal state, the tPM is overdamped and barely visible, whereas below $T_{c}$ it tends to become a pronounced feature as the coherency of the electronic response increases in the superconducting state. The formation of the tPM is typically accompanied by pronounced anomalies of some of the infrared phonon modes. The latter originate from changes in the local electric fields within the intra- and interbilayer regions that are caused by dynamical charging of the $\mathrm{CuO}_{2}$ planes [15]. Accordingly, the anomaly of a phonon mode strongly depends on its frequency and the displacement pattern (i.e., on which ions participate in the vibration).

In underdoped $\mathrm{YBCO}$ the most pronounced SC-induced anomaly occurs for the bending mode at $320 \mathrm{~cm}^{-1}$ that involves mainly vibrations along the $c$ axis of the $\mathrm{O}$ ions within the $\mathrm{CuO}_{2}$ planes [7]. The anomalous behavior of this phonon has been quantitatively reproduced with a relatively simple multilayer model that accounts for the changes in the dynamic local electric fields due to the formation of the tPM $[9,15]$. A corresponding anomaly of a mode at $190 \mathrm{~cm}^{-1}$ (denoted in the following as the $\mathrm{Y}$ mode), which exhibits a sudden narrowing and increases in oscillator strength below $T_{c}$, can also be qualitatively explained with this multilayer model. A quantitative modeling, however, is difficult since it requires precise knowledge of the displacement pattern. Note that the mode involves vibrations of both intrabilayer ions $(\mathrm{Y})$ and interbilayer ones (chain $\mathrm{Cu}$ and $\mathrm{O}$ ) [16].

Notably, a detailed study of the temperature and doping dependence of the tPM and the related anomaly of the $320 \mathrm{~cm}^{-1}$ phonon mode has provided evidence for precursor SC pairing correlations that set in at temperatures well above the bulk superconducting critical temperature $T_{c}$. The bilayer unit acts here as a high-frequency resonator with an eigenfrequency of about 300 to $500 \mathrm{~cm}^{-1}$ (or 10 to $15 \mathrm{THz}$ ) that is sensitive even to short-range and fast-fluctuating SC correlations. Especially, the phonon anomalies, due to the local-electric-field effect caused by the tPM, are very sensitive to the coherency of the intrabilayer response and thus the local SC pairing. Moreover, it was shown that large magnetic fields suppress this onset of coherency, which excludes alternative explanations in terms of charge-density-wave or antiferromagnetic correlations that would be rather enhanced or hardly affected [10].

\section{B. $\boldsymbol{c}$-axis response of pnictides}

The iron arsenide high- $T_{c}$ superconductors, which were discovered in 2008, have a layered structure similar to that of the cuprates $[17,18]$. Their FeAs layers are separated either by monolayers of atoms of alkali, alkaline-earth, or rare-earth metals or by thicker, insulating layers containing oxygen and/or fluorine. This raises the question of whether similar spectroscopic features, as described above for the cuprates, occur in the $c$-axis response of these iron arsenides.

To date, only a few reports of the $c$-axis response of the iron arsenides and selenides exist. The available single crystals are typically rather small or cleave very easily, making it difficult to obtain ac surfaces of the size and quality required for infrared spectroscopy. To the best of our knowledge, reports on the $c$-axis conductivity of single crystals exist only for the undoped parent compound Ba-122 and for $\mathrm{Ba}_{0.6} \mathrm{~K}_{0.4} \mathrm{Fe}_{2} \mathrm{As}_{2}$ (BKFA) around optimum doping with $T_{c}=39 \mathrm{~K}[19,20]$. In both cases a bad-metal-like $c$-axis conductivity with an extremely broad Drude band was observed in the normal state, with no clear sign of a spin density wave (SDW) or SC gap below $T_{N}=135 \mathrm{~K}$ and $T_{c}=39 \mathrm{~K}$, respectively. Whereas the absence in the $c$-axis response of the high-energy SDW gap feature occurring in the in-plane response of Ba-122 can be understood in terms of the in-plane-oriented wave vector of the SDW order, the lack of a clear SC gap feature in the $c$-axis response of BKFA is rather puzzling since it contrasts with the in-plane response for which an almost complete suppression of the optical conductivity has been observed at $\omega \leqslant 2 \Delta_{S C} \simeq 150 \mathrm{~cm}^{-1}$ that is characteristic of a (or several) nearly isotropic gap(s). These data could be reconciled only by assuming that the electronic bands and/or Brillouin-zone segments contributing to the in-plane ( $c$-axis) transport possess large (small) SC gaps. At present, it is not clear whether calculations of the electronic band structure support such a scenario. Moreover, to the best of our knowledge, no information exists yet on the $c$-axis response of underdoped iron arsenide samples for which evidence of a pseudogap effect in terms of a partial suppression of low-energy states that sets in already well above $T_{c}$ has been reported from studies of the in-plane response [21]. On the other hand, an incoherent $c$-axis response similar to that of underdoped cuprates was reported for the iron pnictide compound $\mathrm{FeTe}_{0.55} \mathrm{Se}_{0.45}$ in Ref. [22].

\section{C. $c$-axis response from polycrystalline samples}

Valuable information about the $c$-axis response of layered materials, like the cuprates and iron arsenides, can also be obtained from infrared spectroscopy on polycrystalline samples. This was already demonstrated for the cuprates, for which it was shown that the dominant features in the measured reflectivity spectrum $R_{\text {poly }}$ arise from structures due to the IR-active phonon modes and electronic plasma modes that occur in the $c$-axis reflectivity $R_{c}$. Due to the strongly metallic in-plane response, the $a b$ component of the far-infrared reflectivity $R_{a b}$ is close to unity and exhibits only weak features due to phonons and/or electronic modes and related gap features that hardly show up in $R_{\text {poly }}$ [23]. The close correspondence of the features in $R_{\text {poly }}$ to the ones in $R_{c}$ as measured directly on the $a c$ surface of single crystals has been demonstrated, e.g., for LSCO and YBCO [4,24-26]. Likewise, measurements of polycrystalline Sm,Nd-1111 have already provided information about the SC gap features in the $c$-axis response and have suggested that the optical response of this material is strongly anisotropic [27]. Notably, this study revealed a clear SC gap feature with an onset of the suppression of the conductivity at $2 \Delta_{S C} \simeq 300 \mathrm{~cm}^{-1}$ in the $c$-axis 

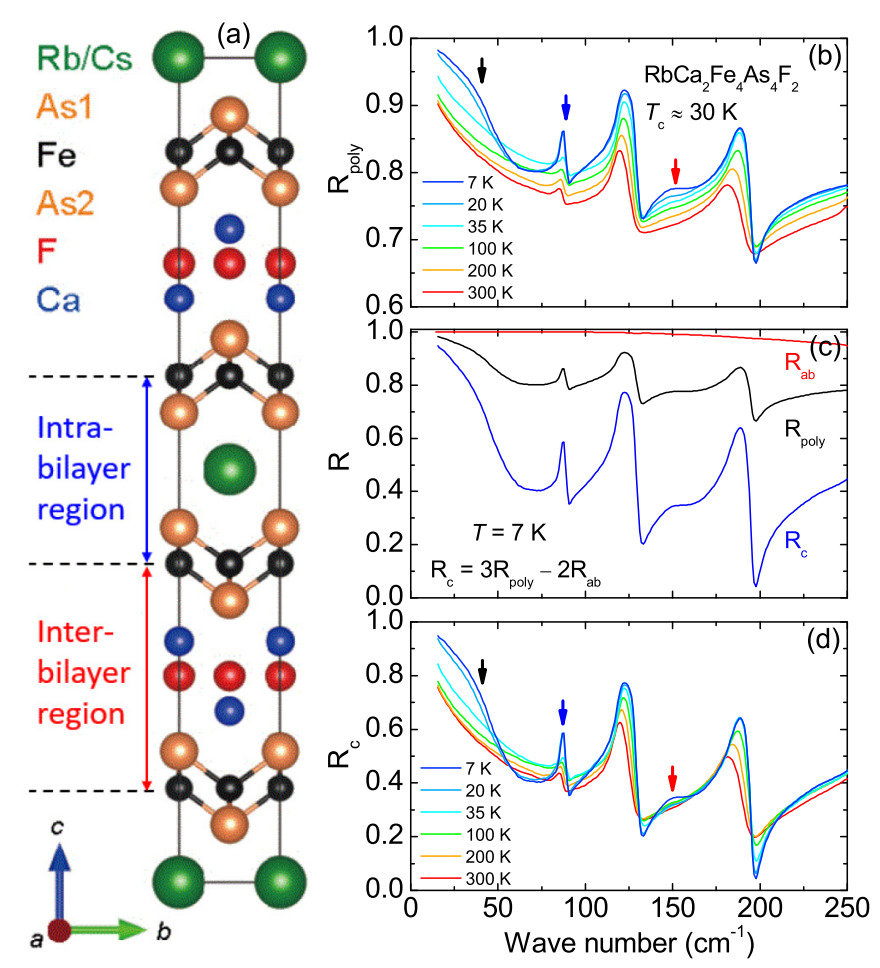

FIG. 1. (a) Sketch of the bilayer-type crystal structure of $A \mathrm{Ca}_{2} \mathrm{Fe}_{4} \mathrm{As}_{4} \mathrm{~F}_{2}(A=\mathrm{Rb}, \mathrm{Cs})$. (b) Far-infrared reflectivity spectra of polycrystalline $\mathrm{RbCa}_{2} \mathrm{Fe}_{4} \mathrm{As}_{4} \mathrm{~F}_{2}$ at several temperatures above and below $T_{c} \simeq 30 \mathrm{~K}$. (c) Comparison of the reflectivity spectrum of the polycrystalline sample $R_{\text {poly }}$, the in-plane spectrum $R_{a b}$ as measured on a corresponding Cs-12442 crystal (from Ref. [31]), and the derived $c$-axis spectrum using $R_{c}=3 R_{\text {poly }}-2 R_{a b}$. (d) Temperature dependence of the derived $c$-axis spectra.

response. However, the gaplike suppression of $\sigma_{1 c}$ is rather gradual and remains incomplete (down to the lowest measured frequency) compared to the in-plane conductivity for which a much sharper gap feature appears that is characteristic of an isotropic SC gap.

Recently, a new iron arsenide superconductor $A \mathrm{Ca}_{2} \mathrm{Fe}_{4} \mathrm{As}_{4} \mathrm{~F}_{2}(A=\mathrm{Rb}, \mathrm{Cs})$ has become available that is very interesting since it has a bilayer-type structure similar to that of the cuprates YBCO and Bi-2212 described above [28-30]. Figure 1(a) shows that it has pairs of FeAs layers that are separated by $\mathrm{Cs}$ or $\mathrm{Rb}$ monolayers that are likely more conducting than the insulator-like $\mathrm{Ca}_{2} \mathrm{~F}_{2}$ double layer that determines the interbilayer conductivity. In the following we show that the $c$-axis response of this bilayer-type iron arsenide superconductor exhibits spectroscopic features that are characteristic of a tPM and phonon anomalies that can be understood in terms of dynamic charging of the FeAs layers and a corresponding modifications of the local electric fields.

\section{EXPERIMENTAL METHOD AND MULTILAYER MODEL}

The growth of polycrystalline samples of (Cs, Rb)-12442 and of corresponding single crystals is described in Ref. [29]. We followed the same procedure to grow additional Ni substituted polycrystalline samples with $1 \%$ and $5 \% \mathrm{Ni}$ for $\mathrm{Fe}$.

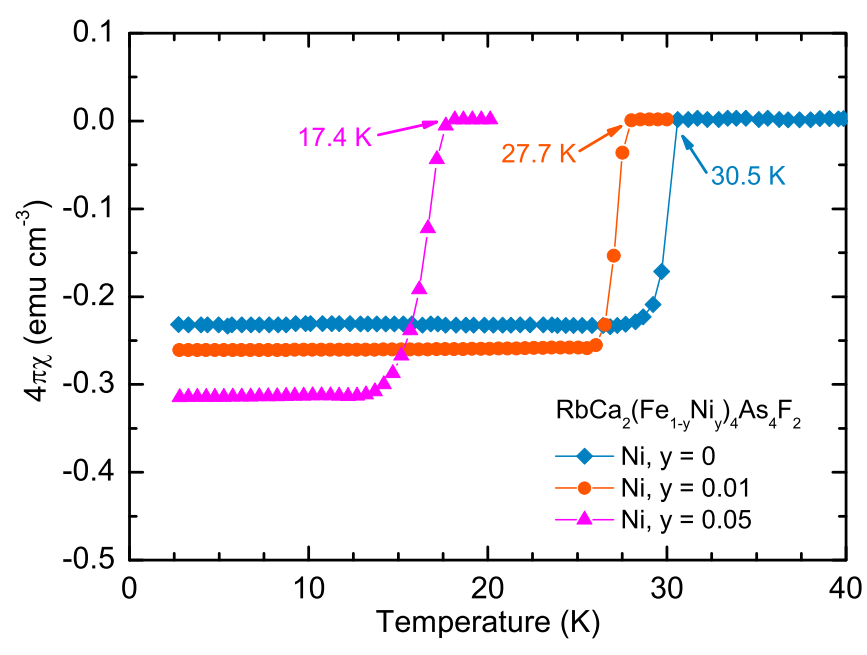

FIG. 2. Temperature dependence of magnetic susceptibility measured at $10 \mathrm{Oe}$ in $\mathrm{FC}$ mode for the bilayer iron-based superconductor $\mathrm{RbCa}_{2}\left(\mathrm{Fe}_{1-y} \mathrm{Ni}_{y}\right)_{4} \mathrm{As}_{4} \mathrm{~F}_{2}$ with $y=0,0.01$, and 0.05 .

The superconducting transition temperatures of these polycrystalline samples of $T_{c} \simeq 30,27$, and $17 \mathrm{~K}$ for $0 \%, 1 \%$, and $5 \% \mathrm{Ni}$, respectively, as obtained from the dc magnetization data are shown in Fig. 2.

The infrared reflectivity study of the in-plane response of a Cs-12442 single crystal is presented in Ref. [31]. We used the same optical setup to obtain the far-infrared reflectivity spectra of the corresponding polycrystalline samples of Rb12442 with $0 \%, 1 \%$, and $5 \% \mathrm{Ni}$. The data were obtained on surfaces of sintered pellets that were polished to optical grade using oil-based diamond paste. Figure 1(a) shows a sketch of the bilayer-type crystal structure of the 12442 compound with the intrabilayer and interbilayer regions indicated by blue and red arrows. Figures 1(b) to 1(d) illustrate the geometrical averaging approach that was used to deduce from the measured reflectivity spectra of the polycrystalline sample $R_{\text {poly }}$ and the in-plane reflectivity spectra of the single crystal $R_{a b}$ the $c$-axis component of the reflectivity according to $R_{\text {poly }}=$ $(1 / 3) R_{c}+(2 / 3) R_{a b}$. Note that this approach is valid only if the size of the crystallites exceeds the effective wavelength of the light, $\lambda / n$, where $\lambda$ is the wavelength in vacuum and $n$ is the refractive index of the material $[32,33]$. Thanks to the very large values of $n$ (especially for the $a b$ component), this condition can be fulfilled for micrometer-sized crystallites, even in the far-infrared (FIR) region with $\lambda \sim 100 \mu \mathrm{m}$. Alternatively, we used an effective medium approximation [32] to analyze the data, as shown in the Appendix. While both approaches yielded qualitatively similar results, we found that the $R_{c}$ spectra obtained from the geometrical averaging were generally in better agreement with the multilayer model. Accordingly, we focus the following discussion on the data obtained with the geometrical averaging approach.

The further analysis of the obtained $R_{c}$ spectra in terms of the dielectric function $\varepsilon_{c}$ with $R_{c}=\left|\frac{1-\sqrt{\varepsilon_{c}}}{1+\sqrt{\varepsilon_{c}}}\right|^{2}$ and $\varepsilon_{c}=\varepsilon+\varepsilon_{\mathrm{ph}}$, where $\varepsilon$ and $\varepsilon_{\mathrm{ph}}$ account for the electronic and phonon contributions, respectively, was performed with the same multilayer model that was previously used for the $c$-axis spectra of single crystals of the bilayer cuprates YBCO and Bi-2212 [9,10]. 
The formula for the electronic dielectric function $\varepsilon$ reads

$$
\varepsilon(\omega)=\frac{\varepsilon_{\mathrm{bl}}(\omega) \varepsilon_{\mathrm{int}}(\omega)}{z_{\mathrm{bl}} \varepsilon_{\mathrm{int}}(\omega)+z_{\mathrm{int}} \varepsilon_{\mathrm{bl}}(\omega)},
$$

where $\varepsilon_{\mathrm{bl}}(\omega)$ and $\varepsilon_{\text {int }}(\omega)$ are the local dielectric functions connected to the two different local conductivities as $\varepsilon_{\mathrm{bl}}(\omega)=$ $\varepsilon_{\infty}+\frac{i \sigma^{\mathrm{bl}}(\omega)}{\omega \epsilon_{0}}$ and $\varepsilon_{\mathrm{int}}(\omega)=\varepsilon_{\infty}+\frac{i \sigma^{\mathrm{git}}(\omega)}{\omega \epsilon_{0}}$ and the factors $z_{\mathrm{bl}}=$ $d_{\mathrm{bl}} /\left(d_{\mathrm{bl}}+d_{\mathrm{int}}\right)$ and $z_{\mathrm{int}}=d_{\mathrm{int}} /\left(d_{\mathrm{bl}}+d_{\mathrm{int}}\right)$ are the volume fractions of the intra- and interbilayer regions, respectively. Here we used $d_{\mathrm{bl}}=7.21 \AA$ and $d_{\mathrm{int}}=8.614 \AA$ for the $\mathrm{Rb}-12442$ samples [29]. The local dielectric functions $\varepsilon_{\mathrm{bl}}(\omega)$ and $\varepsilon_{\text {int }}(\omega)$ are modeled by the following set of Drude-Lorentz oscillators:

$$
\begin{aligned}
\varepsilon_{a}(\omega)= & \varepsilon_{\infty}-\frac{\Omega_{p S, a}^{2}}{\omega(\omega+i \delta)}-\frac{\Omega_{p D, a}^{2}}{\omega^{2}+i \omega \gamma_{D, a}} \\
& +\frac{S_{a}^{2}}{\omega_{0, a}^{2}-\omega^{2}-i \omega \Gamma_{a}}, \quad a \in\{\mathrm{bl}, \text { int }\} .
\end{aligned}
$$

The second and third terms account for the response of the superconducting condensate and the quasiparticles with a finite scattering rate (Drude term), respectively. The fourth term represents an additional Lorentzian oscillator that describes the higher-energy part of the spectra.

\section{RESULTS AND DISCUSSION}

The most important features in the $c$-axis response of $\mathrm{Rb}$ 12442 are indicated with colored arrows in Figs. 1(b) and 1(d). The red arrow marks the tPM around $150 \mathrm{~cm}^{-1}$ that develops below $T_{c} \simeq 30 \mathrm{~K}$ and arises from the out-of-phase electronic response of the intra- and interbilayer regions (as described in the Introduction). The blue arrow highlights an IR-active phonon mode that exhibits a pronounced SC-induced anomaly in terms of a sudden increase of its spectral weight below $T_{c}$. The low frequency of this phonon mode of about $90 \mathrm{~cm}^{-1}$ suggests that it involves the heavy $\mathrm{Rb}$ atoms in the center of the intrabilayer unit [see Fig. 1(a)]. Its displacement pattern (eigenvector) is thus likely similar to that of the $\mathrm{Y}$ mode at $190 \mathrm{~cm}^{-1}$ in YBCO. Notably, this latter Y mode exhibits a similar SC-induced anomaly with a sudden increase of its oscillator strength below $T_{c}$, as was shown in Refs. [7,34,35]. Finally, the black arrow marks the low-frequency plasma edge due to the lowest longitudinal plasma mode, which arises from a weak, Josephson-type coupling of the FeAs layers through the interbilayer regions, i.e., the insulating $\mathrm{Ca}_{2} \mathrm{~F}_{2}$ layers. Notably, the counterparts of all three anomalous lowtemperature features were previously observed in the $c$-axis response of underdoped YBCO.

Figure 3(a) shows the $R_{c}$ spectra (open symbols) at $T=$ $35 \mathrm{~K}$ in the normal state just above $T_{c}$ and at $7 \mathrm{~K} \ll T_{c}$, together with the best fits obtained with the multilayer model (solid lines). It demonstrates that the multilayer model provides a good description of the $c$-axis response obtained from a polycrystalline sample. Note that the contribution of the infrared-active phonon modes to the dielectric function has been fitted using the following function:

$$
\varepsilon_{\mathrm{ph}}(\omega)=\sum_{\mathrm{ph}} \frac{\Omega_{\mathrm{ph}}^{2}-i \omega \beta_{\mathrm{ph}}}{\omega_{0, \mathrm{ph}}^{2}-\omega^{2}-i \omega \gamma_{\mathrm{ph}}},
$$

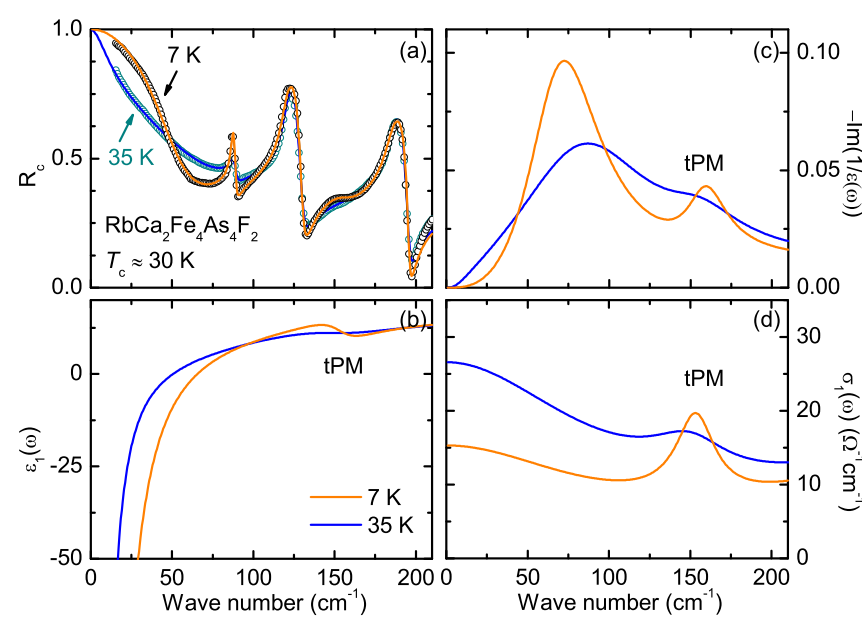

FIG. 3. (a) Fits of the $c$-axis reflectivity spectra with the multilayer model for $\mathrm{RbCa}_{2} \mathrm{Fe}_{4} \mathrm{As}_{4} \mathrm{~F}_{2}$ at $35 \mathrm{~K}$ just above $T_{c}$ and at $7 \mathrm{~K}$ well below $T_{c}$. (b) Real part of the dielectric function, (c) loss function, and (d) optical conductivity at 35 and $7 \mathrm{~K}$ after subtraction of the sharp phonon modes.

which has been added to the expression on the right-hand side of Eq. (1). The sum in Eq. (3) consists of Lorentzian functions, each with a resonance frequency $\omega_{0, \mathrm{ph}}$, a linewidth $\gamma_{\mathrm{ph}}$, and a complex oscillator strength $\Omega_{\mathrm{ph}}^{2}-i \omega \beta_{\mathrm{ph}}$. The imaginary part of the oscillator strength $i \omega \beta_{\mathrm{ph}}$ enables us to describe the line shape of the phonons even if they are asymmetric because they are renormalized by an interaction, for example, with other phonons or the electronic background.

The electronic $c$-axis response functions obtained from the multilayer modeling, after the subtraction of the IR-active phonon modes, are displayed in Figs. 3(b) to 3(d), in terms of the real part of the dielectric function $\varepsilon_{1}(\omega)$, the loss function $-\operatorname{Im}[1 / \varepsilon(\omega)]$, and the real part of the conductivity $\sigma_{1}(\omega)$, respectively. The normal-state spectra at $35 \mathrm{~K}$ reveal a rather weak Drude response with a screened plasma frequency of $\omega_{p, n} / \sqrt{\varepsilon_{\infty}} \approx 70 \mathrm{~cm}^{-1}$ (with $\varepsilon_{\infty}=4$ fixed in the multilayer modeling) and only a very faint and broad peak around $150 \mathrm{~cm}^{-1}$ due to a strongly overdamped tPM. In the SC state at $7 \mathrm{~K}$, the spectral weight of the Drude peak is strongly reduced, and the missing weight is redistributed to a $\delta$ function at zero frequency and to the peak around $150 \mathrm{~cm}^{-1}$, which arises from a strongly enhanced and sharpened tPM.

Figures 4(a) and 4(b) show the spectra of the local conductivities of the intrabilayer and the interbilayer regions, $\sigma_{1}^{\mathrm{bl}}(\omega)$ and $\sigma_{1}^{\text {int }}(\omega)$, respectively, as obtained from the multilayer fitting using Eq. (2). The frequencies of the Lorentzian oscillators were fixed at about $1000 \mathrm{~cm}^{-1}$, and the oscillators are almost temperature independent. The temperature dependencies of the obtained fitting parameters, the plasma frequency and the width of the Drude term, $\Omega_{p D}^{2}$ and $\gamma_{D}$, as well as the weight of the SC $\delta$ function $\Omega_{p S}^{2}$ are displayed in Figs. 4(c) and 4(d) and 4(e) and 4(f) for the intrabilayer and the interbilayer responses, respectively. The values of $\gamma_{D}$ of 40 and $110 \mathrm{~cm}^{-1}$ were obtained by fitting the $7 \mathrm{~K}$ data and kept fixed for higher temperatures. The values of the parameters at several representative temperatures are summarized in Table I. 


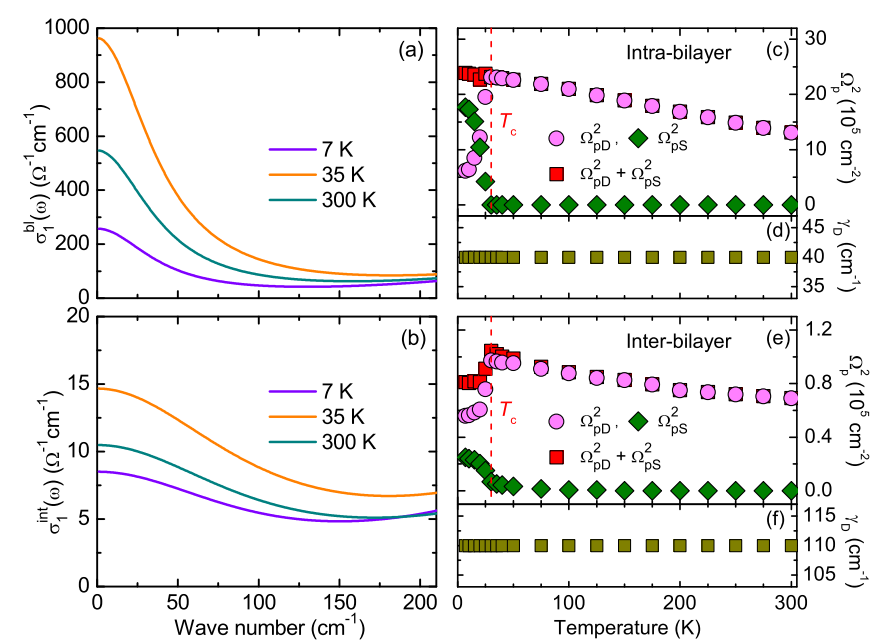

FIG. 4. The local conductivities of (a) the intrabilayer region $\sigma_{1}^{\text {bl }}(\omega)$ and (b) the interbilayer region $\sigma_{1}^{\text {int }}(\omega)$ at 300,35 , and $7 \mathrm{~K}$. Temperature dependences of the obtained values of the fitting parameters, the squared plasma frequency and broadening of the Drude term, $\Omega_{p D}^{2}$ and $\gamma_{D}$, and the squared plasma frequency of the SC condensate $\Omega_{p S}^{2}$ that is proportional to the spectral weight of the SC $\delta$ function for (c) and (d) the intrabilayer and (e) and (f) the interbilayer response, respectively. The $\gamma_{D}$ values of 40 and $110 \mathrm{~cm}^{-1}$, respectively, were obtained by fitting the $7 \mathrm{~K}$ data and were kept fixed for higher temperatures.

At $35 \mathrm{~K}$ in the normal state just above $T_{c}$, this multilayer analysis reveals a rather large, more than 20 -fold difference between the squared plasma frequencies of the intra- and the interbilayer regions (recall that $\Omega_{p D}^{2} \sim n / m^{*}$, where $n$ and $m^{*}$ are the concentration and effective mass of the carriers). Interestingly, the absolute values of $\sigma_{1}^{\mathrm{bl}}(\omega)$ and $\sigma_{1}^{\mathrm{int}}(\omega)$ are similar to the ones reported for underdoped YBCO with $T_{c} \sim$ $60 \mathrm{~K}[9,10]$.

An important difference concerns the spectral shape of the interbilayer conductivity $\sigma_{1}^{\text {int }}(\omega)$, which in underdoped YBCO was found to be insulatorlike with a steady decrease of the absolute values as temperature is reduced (see Fig. 1(d) of Ref. [10]). The insulatorlike behavior of $\sigma_{1}^{\text {int }}(\omega)$ was interpreted in terms of the aforementioned pseudogap phenomenon which depletes the low-energy electronic states in the antinodal parts of the Fermi surface [close to $(0, \pi)$ or $(\pi, 0)$ ] at which the $c$-axis hopping parameter across the interbilayer region $t_{\perp}^{\mathrm{int}}$ is maximal [10].

TABLE I. Values of the parameters $\Omega_{p D}, \gamma_{D}$, and $\Omega_{p s}$ entering Eq. (2) obtained by fitting the $c$-axis reflectivity spectra of $\mathrm{RbCa}_{2} \mathrm{Fe}_{4} \mathrm{As}_{4} \mathrm{~F}_{2}$ for three representative temperatures. All quantities are in units of $\mathrm{cm}^{-1}$.

\begin{tabular}{|c|c|c|c|c|c|c|}
\hline \multirow[b]{2}{*}{$T(\mathrm{~K})$} & \multicolumn{3}{|c|}{ Intrabilayer } & \multicolumn{3}{|c|}{ Interbilayer } \\
\hline & $\Omega_{p D}$ & $\gamma_{D}$ & $\Omega_{p S}$ & $\Omega_{p D}$ & $\gamma_{D}$ & $\Omega_{p S}$ \\
\hline 300 & 786 & 40 & 1330 & 237 & 110 & 158 \\
\hline 35 & 1520 & 40 & 0 & 311 & 110 & 72 \\
\hline 7 & 1146 & 40 & 0 & 263 & 110 & 0 \\
\hline
\end{tabular}
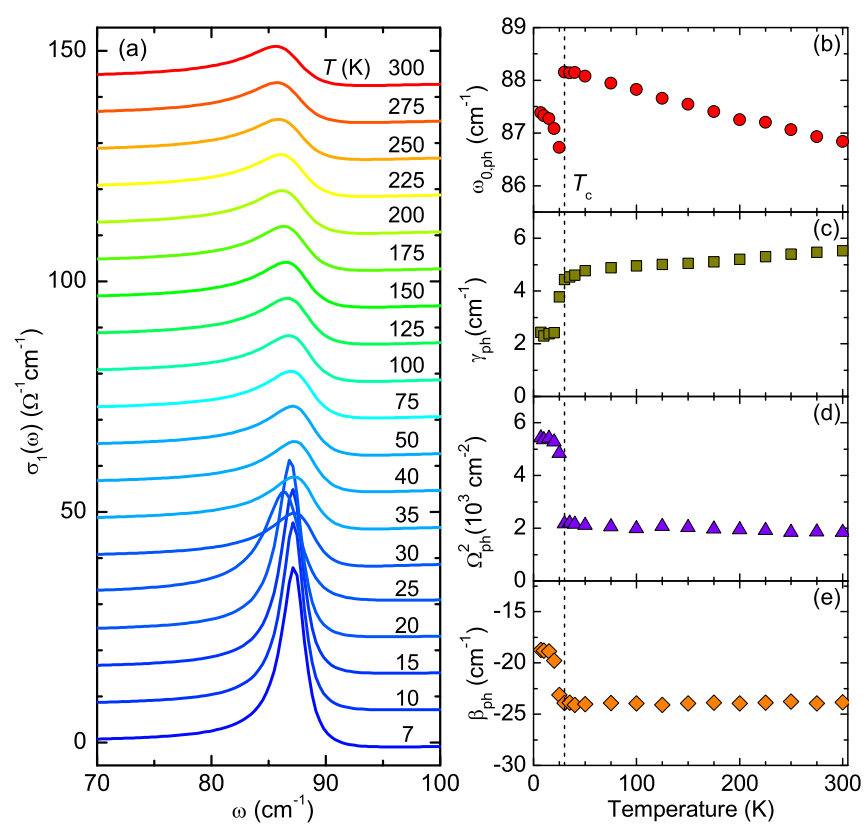

FIG. 5. (a) Temperature dependence of the line shape of the $\mathrm{Rb}$ phonon mode. (b)-(e) Temperature dependences of the resonance frequency $\omega_{0, \mathrm{ph}}$, the linewidth $\gamma_{\mathrm{ph}}$, the oscillator strength $\Omega_{\mathrm{ph}}^{2}$, and the parameter $\beta_{\text {ph }}$ of the phonon as obtained from a fit with an asymmetric Lorentz function. The vertical dashed line denotes the superconducting transition temperature $T_{c}$.

In contrast, for $\mathrm{Rb}-12442$ the interbilayer conductivity $\sigma_{1}^{\text {int }}(\omega)$ is well described in terms of a Drude-type response of itinerant carriers. Moreover, $\sigma_{1}^{\text {int }}(\omega)$ has a metallic temperature dependence and exhibits a nearly twofold increase between 300 and $35 \mathrm{~K}$. It is quite remarkable that the interbilayer response remains coherent, despite the very small absolute value of $\sigma_{1}^{\text {int }}(\omega \rightarrow 0,35 \mathrm{~K}) \simeq 15 \Omega^{-1} \mathrm{~cm}^{-1}$. Certainly, the interbilayer response of the Rb-12442 sample shows no sign of a pseudogap effect similar to the one in the $c$-axis response of the underdoped cuprates.

Concerning the SC-induced changes at $T \ll T_{c} \simeq 30 \mathrm{~K}$, it is evident from Figs. 4(a) and 4(b) that both $\sigma_{1}^{\mathrm{bl}}(\omega)$ and $\sigma_{1}^{\text {int }}(\omega)$ exhibit a clear gaplike suppression of the regular part of the low-energy response; that is, the Drude peak is strongly reduced, and the missing spectral weight is mostly transferred to the $\delta$ function at zero frequency that represents the loss-free response of the SC condensate. For the intrabilayer response $\sigma_{1}^{\mathrm{bl}}(\omega)$ the spectral weight loss of the Drude peak amounts to about $70 \%$ and is fully balanced by the corresponding spectral weight gain of the SC condensate, as shown in Fig. 4(c). For the interbilayer conductivity $\sigma_{1}^{\text {int }}(\omega)$ the spectral weight loss of the Drude peak is only about $40 \%$, and as shown in Fig. 4(e), it is not fully compensated by the spectral weight of the $\delta$ function at zero frequency. The corresponding drop in the total electronic spectral weight $S_{\text {total }}=\frac{d_{\mathrm{bl}}}{d_{\mathrm{bl}}+d_{\mathrm{int}}} S_{\mathrm{bl}}+\frac{d_{\text {int }}}{d_{\mathrm{bl}}+d_{\text {int }}} S_{\text {int }}$ [36] is, to a large extent, compensated by an increase of the phononic spectral weight. Here $S_{\text {total }}, S_{\mathrm{bl}}$, and $S_{\text {int }}$ are the optical spectral weights connected to the total electronic conductivity, $\sigma^{\mathrm{bl}}$, and $\sigma^{\text {int }}$, respectively. Figure 5 shows that the low-frequency mode at $90 \mathrm{~cm}^{-1}$ exhibits, indeed, a significant spectral weight increase below 
$T_{c}$. Taking into account also a similar spectral weight gain below $T_{c}$ of the mode at $120 \mathrm{~cm}^{-1}$ (data are not shown), we estimate that this can almost explain the drop in the electronic spectral weight. The remaining inconsistency is likely due to the uncertainty of fitting the contribution of the SC condensate to $\sigma_{1}^{\text {int }}(\omega)$, which strongly depends on the low-frequency part of the experimental data $\left(\omega<50 \mathrm{~cm}^{-1}\right)$ with sizable error bars.

Overall, the $c$-axis response of this bilayer compound exhibits quite a sizable suppression of the Drude response due to the SC gap formation. The suppression of the low-frequency conductivity is not quite as strong as for the in-plane response of a single crystal, for which the gap(s) appear(s) to be almost isotropic and complete with almost zero conductivity at $\omega<$ $2 \Delta \approx 110 \mathrm{~cm}^{-1}$ [31]. Nevertheless, it is much stronger than the one reported for the $c$-axis response of an optimally doped BKFA single crystal [20] where no clear sign of a SC gap was observed. Note that the weaker signature of the SC gap in the interbilayer response can be explained provided that the band that is most dispersive along the $c$-axis direction has a smaller SC gap than the bands that are more dispersive along the in-plane direction. This conjecture agrees with the angle-resolved photoemission spectroscopy (ARPES) data in Fig. 2 of Ref. [37] which reveal that the $\alpha$ and $\beta$ bands with larger gaps of $2 \Delta \approx 8-16 \mathrm{meV}$ have a more pronounced in-plane dispersion than the $\gamma$ band with a smaller gap $2 \Delta \approx$ $4 \mathrm{meV}$ that has the strongest $c$-axis dispersion.

$\mathrm{Next}$, we discuss the SC-induced anomaly of the Rbphonon mode around $90 \mathrm{~cm}^{-1}$ that is shown in Fig. 5(a). The corresponding temperature dependences of the parameters obtained from fitting the $R_{c}$ spectra with Eq. (3) are displayed in Figs. 5(b) to 5(e). All parameters exhibit clear anomalies that start right below $T_{c} \simeq 30 \mathrm{~K}$. It was previously shown for the underdoped cuprates that the phonon anomalies are particularly sensitive to the onset of local SC pairing interactions at $T>T_{c}$ [10]. The sharp onset of the anomaly of the $\mathrm{Rb}$ mode right below $T_{c} \simeq 30 \mathrm{~K}$ is therefore a clear indication that such a precursor SC pairing does not occur in $\mathrm{Rb}-12442$. This conclusion is supported by the equally sharp anomaly right below $T_{c}$ of the tPM and the related $\delta$ function at zero frequency in $\sigma_{1}^{\text {bl }}$ [see Figs. 4(a) and 4(c)]. As already discussed in the previous paragraph, the multilayer fitting of the $\delta$ function in $\sigma_{1}^{\text {int }}$ has a large uncertainty; its very weak onset slightly above $T_{c}$ is therefore likely an artifact.

In the following, we address possible connections between the tPM feature and the bilayer splitting, i.e., the splitting between the bonding and antibonding bands of the bilayer unit. For the cuprates it was recently shown, based on the multilayer modeling of the $c$-axis response, that such bilayer splitting is detectable above a hole doping of $p \approx 0.10$, where it increases in magnitude towards optimum doping up to 30 $60 \mathrm{meV}[38,39]$. Specifically, it was shown that significantly better fits are obtained if $\sigma_{1}^{\mathrm{bl}}(\omega)$ is described by a Lorentzian function with a peak at an energy comparable to the magnitude of the bilayer splitting, rather than by a Drude response with a peak at zero energy. In the normal state, this Lorentzian peak accounts for the interband transition between the bonding and antibonding bands of the $\mathrm{CuO}_{2}$ bilayer unit that is strongly damped. In the superconducting state, it corresponds to a collective mode that is only weakly damped if the gap $2 \Delta$ is larger than the bilayer splitting [40]. Otherwise, if $2 \Delta$ is smaller than the bilayer splitting, this mode is also strongly damped, and the tPM feature and the phonon anomalies due to the related local electric field effects are expected to be weak. For the present Rb-12442 sample, we find no evidence of such a bilayer splitting of an energy scale larger than about $5 \mathrm{meV}$; that is, we verified that describing $\sigma_{1}^{\mathrm{bl}}(\omega)$ by a Lorentzian function with a peak energy above $5 \mathrm{meV}$ (instead of the Drude function shown in Fig. 4) does not improve the quality of the multilayer fitting. Note that these multilayer fits are not expected to be sensitive to a bilayer splitting smaller than the width of the Drude function of $\sim 5 \mathrm{meV}$. A similarly small bilayer splitting was, indeed, observed in a very recent ARPES study of a K-12442 crystal which has the same type of bilayer structure [37]. As shown in Fig. 2 of [37], a clear bilayer splitting is observed for the holelike $\gamma$ and $\beta$ bands near the Brillouin-zone center. The largest splitting near the Fermi surface occurs for the $\gamma$ band, for which it is in the range of 5 to $10 \mathrm{meV}$, slightly larger than the reported value of the SC gap of the $\gamma$ band of $2 \Delta \approx$ $4 \mathrm{meV}$ [37]. This raises the question of how it is possible that a reasonably well defined tPM appears in the spectra. A likely scenario is the following: The formation of relatively large gaps on the $\alpha$ and $\beta$ bands $(2 \Delta \approx 8-16 \mathrm{meV}$ ) leads to a dramatic sharpening of the bilayer split $\gamma$ bands below $T_{c}$. This effect can, indeed, be seen in Fig. 3 of Ref. [37]. As a consequence, the corresponding low-energy optical transition also sharpens considerably, giving rise to a clear tPM feature. The very low energy of the transition allows one to use the phenomenological multilayer model in its simplest form when analyzing the data, as we did in the previous paragraphs.

Another important issues is that Rb-12442 is expected to be strongly hole doped and located on the overdoped rather than on the underdoped side of the phase diagram. The hole doping of the FeAs layers of Rb-12442 is nominally the same as in $\mathrm{Ba}_{0.5} \mathrm{~K}_{0.5} \mathrm{Fe}_{2} \mathrm{As}_{2}$ [41-43]. Accordingly, it is not too surprising that there is no pseudogap effect above $T_{c}$ due to competing electronic and/or magnetic orders and no onset of local SC pairing at a temperature significantly higher than $T_{c}$.

Accordingly, we have also studied a series of polycrystalline, Ni-substituted samples $\mathrm{RbCa}_{2}\left(\mathrm{Fe}_{(1-y)} \mathrm{Ni}_{y}\right)_{4} \mathrm{As}_{4} \mathrm{~F}_{2}$ with $y=0.01$ and 0.05 for which the hole doping is progressively reduced toward the underdoped regime. It was previously shown in $\mathrm{BaFe}_{2-y} \mathrm{Ni}_{2 y} \mathrm{As}_{2}$ that $\mathrm{Ni}$ substitution gives rise to electron doping at a rate that is about two times faster than for Co substitution [44-46]. It was also shown for $\mathrm{Ba}_{1-x} \mathrm{~K}_{x} \mathrm{Fe}_{2-y}(\mathrm{Co}, \mathrm{Ni})_{y} \mathrm{As}_{2}$ that codoping with $\mathrm{Co}$ or $\mathrm{Ni}$ counteracts the hole doping due to the $\mathrm{K}$ substitution [47]. Moreover, for $\mathrm{CaKFe}_{4-4 y} \mathrm{Ni}_{4 y} \mathrm{As}_{4}$, which at $y=0$ has the same nominal hole doping as Rb-12442, it has already been demonstrated that $\mathrm{Ni}$ substitution allows one to reach the underdoped side of the phase diagram and induce a magnetic order (and possibly also nematic and orbital orders) that coexists and competes with SC $[48,49]$.

The magnetization data of our polycrystalline samples in Fig. 2 confirm that the Ni substitution gives rise to a strong suppression of the SC transition temperature with $T_{c}$ values of 30,27 , and $17 \mathrm{~K}$ for $y=0,0.01$, and 0.05 , respectively. Figures 6(a) and 6(b) show the temperature dependence of the far-infrared spectra of the as-measured reflectivity $R_{\text {poly }}$ 


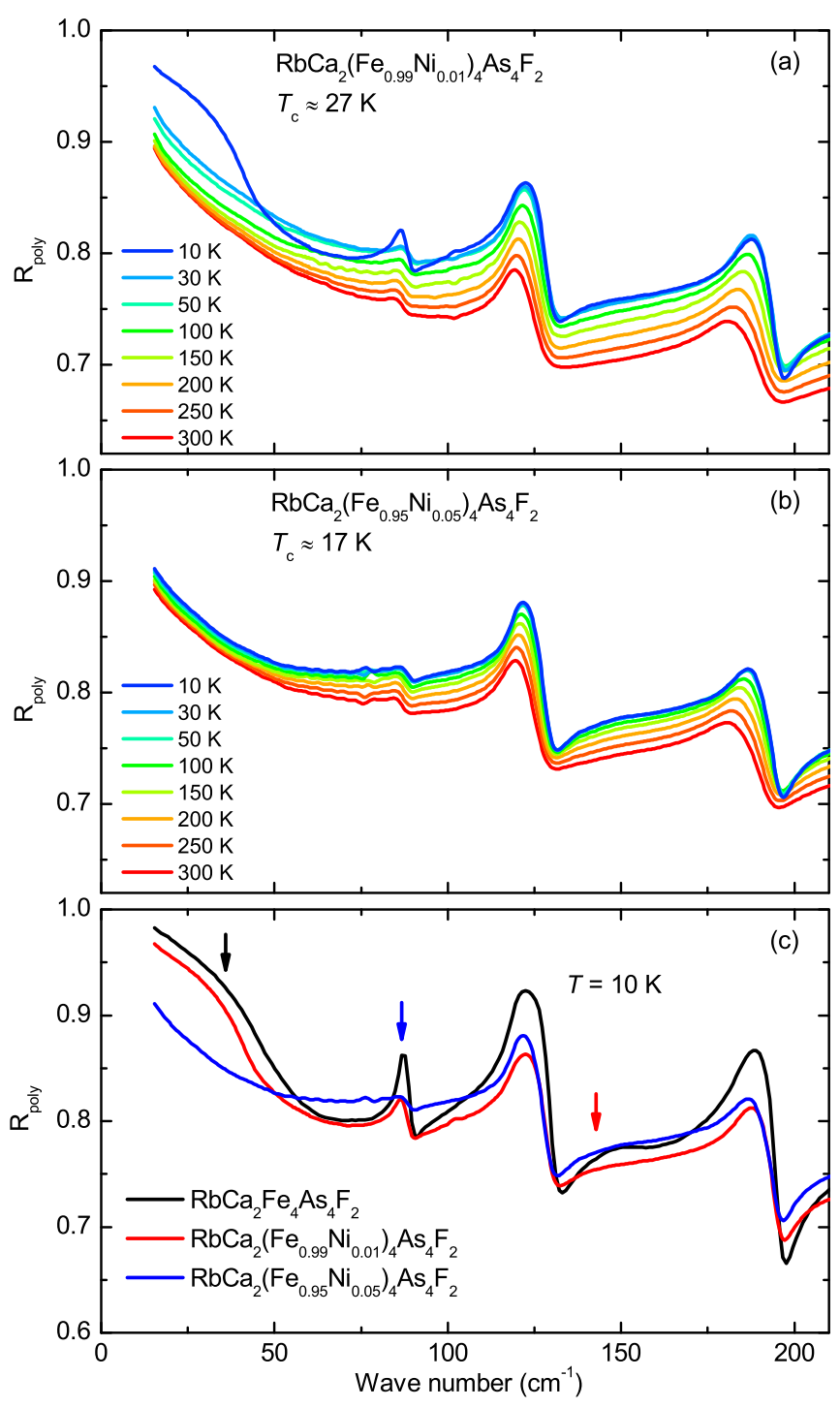

FIG. 6. (a) and (b) Temperature-dependent far-infrared reflectivity spectra of polycrystalline $\mathrm{RbCa}_{2}\left(\mathrm{Fe}_{(1-y)} \mathrm{Ni}_{y}\right)_{4} \mathrm{As}_{4} \mathrm{~F}_{2}$ with $y=$ 0.01 and 0.05 . (c) Comparison of the reflectivity spectra at $10 \mathrm{~K}$ of $\mathrm{RbCa}_{2}\left(\mathrm{Fe}_{(1-y)} \mathrm{Ni}_{y}\right)_{4} \mathrm{As}_{4} \mathrm{~F}_{2}$ with $y=0,0.01$, and 0.05 .

for the polycrystalline samples with $y=0.01$ and 0.05 , respectively. Figure 6(c) compares the spectra at $T=10 \mathrm{~K}<$ $T_{c}$ for the series with $y=0,0.01$, and 0.05 . The characteristic superconductivity-induced features due to the tPM around 150 $\mathrm{cm}^{-1}$, the anomaly of the $\mathrm{Rb}$ phonon mode around $90 \mathrm{~cm}^{-1}$, and the low-frequency plasma edge around $40 \mathrm{~cm}^{-1}$ (that is determined by $\Omega_{p S}^{\text {int }}$ ) are marked by the same kind of arrows as in Fig. 1(b). Figure 6 reveals that these SC-induced anomalies are significantly weakened for the sample with $y=0.01$ and almost absent for $y=0.05$. Moreover, Figs. 6(a) and 6(b) establish that the Ni substitution does not give rise to any anomalous changes of the spectra in the normal state above $T_{c}$, which could be associated either with a pseudogap effect due to a competing order or with precursor pairing correlations above $T_{c}$.

Figures 7(a) and 7(b) show the FIR spectra of $R_{c}$ at 35 and $10 \mathrm{~K}$ (open symbols) at $y=0.01$ and 0.05 , respectively,
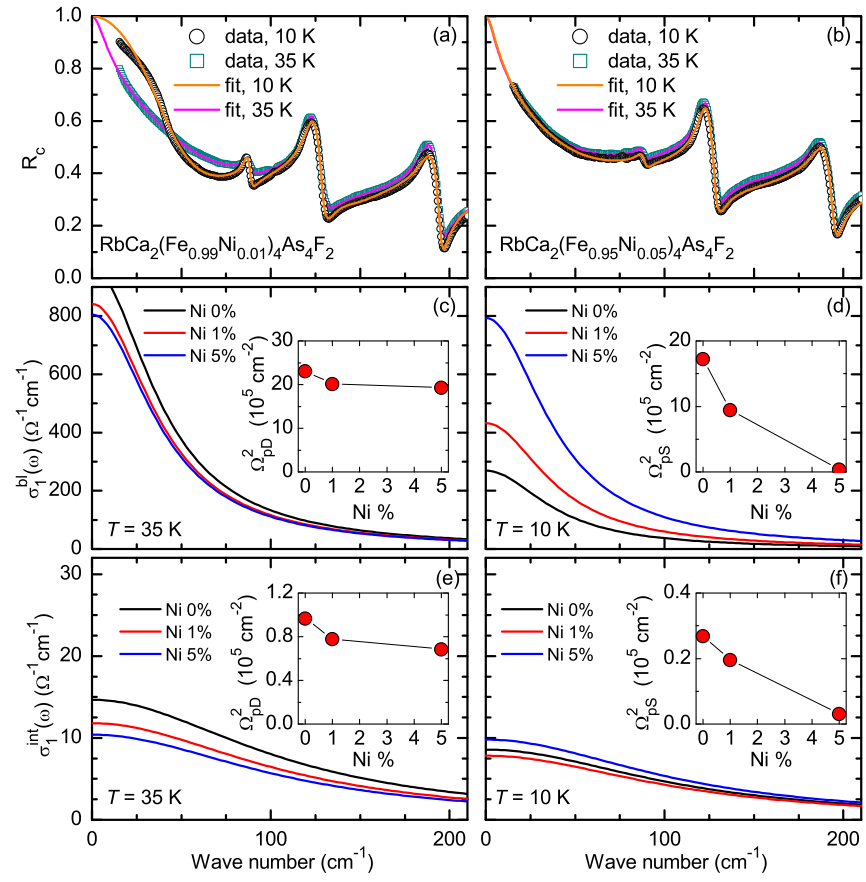

FIG. 7. (a) and (b) Fits of the $c$-axis reflectivity spectra with the multilayer model for $\mathrm{RbCa}_{2}\left(\mathrm{Fe}_{(1-y)} \mathrm{Ni}_{y}\right)_{4} \mathrm{As}_{4} \mathrm{~F}_{2}$ with $y=0.01$ and 0.05 at $35 \mathrm{~K}$ just above $T_{c}$ and $7 \mathrm{~K}$ well below $T_{c}$. (c)-(f) Comparison of the local conductivities of the intrabilayer region $\sigma_{1}^{\mathrm{bl}}(\omega)$ and the interbilayer region $\sigma_{1}^{\text {int }}(\omega)$ for $\mathrm{RbCa}_{2}\left(\mathrm{Fe}_{(1-y)} \mathrm{Ni}_{y}\right)_{4} \mathrm{As}_{4} \mathrm{~F}_{2}$ with $y=0$, 0.01 , and 0.05 at representative temperatures of 35 and $7 \mathrm{~K}$. The insets show the evolution of the corresponding normal-state and SC plasma frequencies as a function of the $\mathrm{Ni}$ concentration.

that were derived using the same geometrical averaging as discussed above and shown in Fig. 1 for $y=0$. For the inplane spectra we used the data for the undoped single crystal from Ref. [31]. The solid lines represent the best fits obtained with the multilayer model. Finally, Figs. 7(c)-7(f) show a comparison of the local conductivities $\sigma_{1}^{\text {bl }}$ and $\sigma_{1}^{\text {int }}$ at 35 and $10 \mathrm{~K}$. The insets show the evolution of the corresponding normal state and SC plasma frequencies as a function of the $\mathrm{Ni}$ content. They reveal that the Ni substitution hardly affects the normal-state conductivity and the related plasma frequency $\Omega_{p D}$, whereas it gives rise to a strong suppression of the SC $\delta$ function and $\Omega_{p s}$.

\section{SUMMARY AND CONCLUSION}

With infrared spectroscopy we have studied Rb-12442 with $y=0,0.01$, and 0.05 , which has a bilayer-type structure similar to the cuprates, like YBCO and Bi2212. From combined measurements of polycrystalline samples and the in-plane response of corresponding single crystals and using a geometrical averaging approach, we have derived the $c$-axis conductivity of these samples. In analogy to the cuprates, the $c$-axis response exhibits several spectroscopic features that are characteristic of a layered structure with very different strengths of the electronic response of the intra- and interbilayer regions. In particular, we find that a transverse plasma mode develops around $150 \mathrm{~cm}^{-1}$ that is strongly overdamped in the normal state but sharpens and is strongly enhanced 
below $T_{c} \simeq 30 \mathrm{~K}$. Along with the tPM comes a pronounced SC-induced anomaly of the lowest IR-active phonon mode around $90 \mathrm{~cm}^{-1}$ that involves the displacement of the heavy $\mathrm{Rb}$ ions and arises from the local-electric-field effects caused by the dynamical charging of the FeAs layers connected to the formation of the tPM. Moreover, we observed a pronounced low-frequency plasma edge (around $50 \mathrm{~cm}^{-1}$ ) that is characteristic of a very weak electronic interbilayer response. Using a multilayer model similar to the one previously used for the $c$-axis response of underdoped YBCO, we achieved a good description of the experimental data. The obtained values of the inter- and intrabilayer conductivities are comparable to the ones of underdoped YBCO with $T_{c}=60 \mathrm{~K}$. A marked difference concerns the absence of a pseudogap effect in the interbilayer conductivity of $\mathrm{Rb}-12442$, which, despite a very small absolute value of $\sigma_{1}^{\text {int }}(\omega \rightarrow 0) \simeq 15 \Omega^{-1} \mathrm{~cm}^{-1}$, remains metal-like. Moreover, from the temperature dependence of the tPM and the anomaly of the Rb phonon mode, we obtained no evidence of the onset of precursor SC pairing correlations well above the bulk SC transition temperature $T_{c}$ at which the phase coherence becomes macroscopic. Such an onset is also not observed for the Ni-substituted sample for which the hole doping is reduced toward the underdoped regime. This is despite a strong Ni-induced suppression of the plasma frequency of the SC $\delta$ function.

Our observations suggest that the pseudogap and the onset of local superconducting pairing correlations well above $T_{c}$ are unique features of the cuprates; that is, they are not just the result of a strong electronic anisotropy due to a very weak interlayer conductivity. The multilayer analysis also reveals clear signatures of the SC gap formation in the inter- and intrabilayer conductivities that are much more pronounced than in the previous report on a BKFA crystal without such a bilayer structure [20]. A sizable, ungapped part of the low-frequency conductivity, which is more pronounced (related to the overall strength of the response) for the interbilayer than for the intrabilayer conductivity, can be understood in terms of the multiband structure with a $\gamma$ band that has a rather small gap and is more dispersive along the $c$ axis than the other bands with larger gaps that disperse more strongly in the $k_{x}-k_{y}$ plane. Such different dispersion behaviors and gap magnitudes were, indeed, recently reported in an ARPES study of an isostructural K-12442 single crystal [37].

\section{ACKNOWLEDGMENTS}

Work at the University of Fribourg was supported by the Schweizer Nationalfonds (SNF) by Grant No. 200020172611. D.M. and A.D were supported by the MEYS of the Czech Republic under the project CEITEC 2020 (Grant No. LQ1601) and by the Czech Science Foundation (GAČR) under Project No. GA20-10377S.
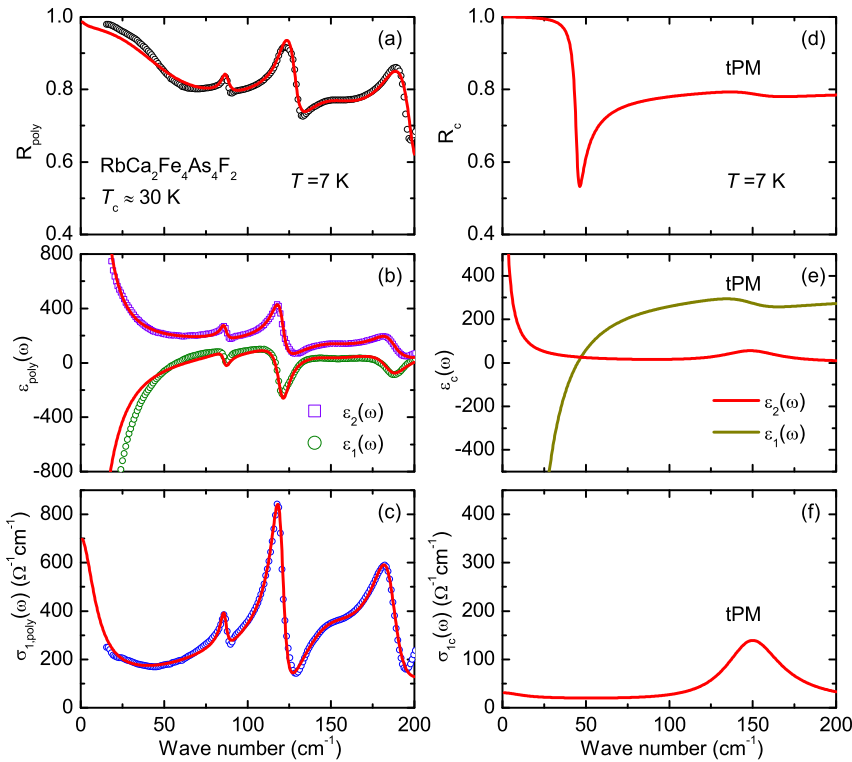

FIG. 8. Fits of (a) the reflectivity, (b) the dielectric function, and (c) the real part of optical conductivity spectra with the effective medium approximation for polycrystalline $\mathrm{RbCa}_{2} \mathrm{Fe}_{4} \mathrm{As}_{4} \mathrm{~F}_{2}$ at $7 \mathrm{~K}$. The $c$-axis response of (d) the reflectivity, (e) the dielectric function, and (f) the real part of the optical conductivity at $7 \mathrm{~K}$ after subtraction of the sharp phonon modes.

\section{APPENDIX: EFFECTIVE MEDIUM APPROXIMATION}

According to the Maxwell-Garnett theory, the effective dielectric properties $\varepsilon_{\text {eff }}$ of a polycrystalline material are determined by the following equation:

$$
\varepsilon_{\mathrm{eff}}=\varepsilon_{a b} \frac{\left(\varepsilon_{c}+2 \varepsilon_{a b}\right)+2 p\left(\varepsilon_{c}-\varepsilon_{a b}\right)}{\left(\varepsilon_{c}+2 \varepsilon_{a b}\right)-p\left(\varepsilon_{c}-\varepsilon_{a b}\right)},
$$

where the effective medium consists of a matrix with $\varepsilon_{a b}$ ( $a b$-plane response) and inclusions with $\varepsilon_{c}$ ( $c$-axis response) and $p$ corresponds to the volume fraction of the inclusions in the effective medium. The effective medium approximation dielectric function is fit to the data for polycrystalline $\mathrm{RbCa}_{2} \mathrm{Fe}_{4} \mathrm{As}_{4} \mathrm{~F}_{2}$, where $\varepsilon_{a b}$ is described by a simple Drude model, $\varepsilon_{c}$ is described by the multilayer model mentioned in the main text, and the volume fraction $p=1 / 3$. Figures 8 (a)8(c) show the fitting results to the reflectivity, the dielectric function, and the real part of optical conductivity of polycrystalline $\mathrm{RbCa}_{2} \mathrm{Fe}_{4} \mathrm{As}_{4} \mathrm{~F}_{2}$ at $7 \mathrm{~K}$, respectively. The corresponding electronic $c$-axis response functions obtained from the effective medium approximation, after the subtraction of the IR-active phonon modes, are displayed in Figs. 8(d) to 8(f) in terms of the reflectivity $R_{c}$, the dielectric function $\varepsilon_{c}(\omega)$, and the real part of the conductivity $\sigma_{1 c}(\omega)$, respectively. Qualitatively, the effective medium approximation approach yielded results similar to the geometrical averaging approach we discussed in the main text.
[1] D. N. Basov and T. Timusk, Rev. Mod. Phys. 77, 721 (2005).

[2] D. N. Basov, T. Timusk, B. Dabrowski, and J. D. Jorgensen, Phys. Rev. B 50, 3511 (1994).
[3] S. Tajima, J. Schützmann, S. Miyamoto, I. Terasaki, Y. Sato, and R. Hauff, Phys. Rev. B 55, 6051 (1997). 
[4] C. C. Homes, T. Timusk, R. Liang, D. A. Bonn, and W. N. Hardy, Phys. Rev. Lett. 71, 1645 (1993).

[5] T. Timusk and B. Statt, Rep. Prog. Phys. 62, 61 (1999).

[6] A. V. Puchkov, D. N. Basov, and T. Timusk, J. Phys.: Condens. Matter 8, 10049 (1996).

[7] C. Bernhard, D. Munzar, A. Golnik, C. T. Lin, A. Wittlin, J. Humlíček, and M. Cardona, Phys. Rev. B 61, 618 (2000).

[8] A. V. Boris, D. Munzar, N. N. Kovaleva, B. Liang, C. T. Lin, A. Dubroka, A. V. Pimenov, T. Holden, B. Keimer, Y.-L. Mathis, and C. Bernhard, Phys. Rev. Lett. 89, 277001 (2002).

[9] A. Dubroka, L. Yu, D. Munzar, K. Kim, M. Rössle, V. Malik, C. Lin, B. Keimer, T. Wolf, and C. Bernhard, Eur. Phys. J. Spec. Top. 188, 73 (2010).

[10] A. Dubroka, M. Rössle, K. W. Kim, V. K. Malik, D. Munzar, D. N. Basov, A. A. Schafgans, S. J. Moon, C. T. Lin, D. Haug, V. Hinkov, B. Keimer, T. Wolf, J. G. Storey, J. L. Tallon, and C. Bernhard, Phys. Rev. Lett. 106, 047006 (2011).

[11] A. Damascelli, Z. Hussain, and Z.-X. Shen, Rev. Mod. Phys. 75, 473 (2003).

[12] E. Uykur, K. Tanaka, T. Masui, S. Miyasaka, and S. Tajima, Phys. Rev. Lett. 112, 127003 (2014).

[13] V. Železný, S. Tajima, D. Munzar, T. Motohashi, J. Shimoyama, and K. Kishio, Phys. Rev. B 63, 060502(R) (2001).

[14] D. van der Marel and A. Tsvetkov, Czech. J. Phys. 46, 3165 (1996).

[15] D. Munzar, C. Bernhard, A. Golnik, J. Humlíček, and M. Cardona, Solid State Commun. 112, 365 (1999).

[16] J. Humlíček, A. Litvinchuk, W. Kress, B. Lederle, C. Thomsen, M. Cardona, H.-U. Habermeier, I. Trofimov, and W. König, Phys. C (Amsterdam, Neth.) 206, 345 (1993).

[17] J. Paglione and R. L. Greene, Nat. Phys. 6, 645 (2010).

[18] D. N. Basov and A. V. Chubukov, Nat. Phys. 7, 272 (2011).

[19] Z. G. Chen, T. Dong, R. H. Ruan, B. F. Hu, B. Cheng, W. Z. Hu, P. Zheng, Z. Fang, X. Dai, and N. L. Wang, Phys. Rev. Lett. 105, 097003 (2010).

[20] B. Cheng, Z. G. Chen, C. L. Zhang, R. H. Ruan, T. Dong, B. F. Hu, W. T. Guo, S. S. Miao, P. Zheng, J. L. Luo, G. Xu, P. Dai, and N. L. Wang, Phys. Rev. B 83, 144522 (2011).

[21] S. J. Moon, A. A. Schafgans, S. Kasahara, T. Shibauchi, T. Terashima, Y. Matsuda, M. A. Tanatar, R. Prozorov, A. Thaler, P. C. Canfield, A. S. Sefat, D. Mandrus, and D. N. Basov, Phys. Rev. Lett. 109, 027006 (2012).

[22] S. J. Moon, C. C. Homes, A. Akrap, Z. J. Xu, J. S. Wen, Z. W. Lin, Q. Li, G. D. Gu, and D. N. Basov, Phys. Rev. Lett. 106, 217001 (2011).

[23] Z. Schlesinger, R. T. Collins, M. W. Shafer, and E. M. Engler, Phys. Rev. B 36, 5275 (1987).

[24] D. A. Bonn, J. E. Greedan, C. V. Stager, T. Timusk, M. G. Doss, S. Herr, K. Kamarás, C. D. Porter, D. B. Tanner, J. M. Tarascon, W. R. McKinnon, and L. H. Greene, Phys. Rev. B 35, 8843(R) (1987).

[25] D. A. Bonn, J. E. Greedan, C. V. Stager, T. Timusk, M. G. Doss, S. L. Herr, K. Kamarás, and D. B. Tanner, Phys. Rev. Lett. 58, 2249 (1987).

[26] S. Uchida, K. Tamasaku, and S. Tajima, Phys. Rev. B 53, 14558 (1996).

[27] A. Dubroka, K. W. Kim, M. Rössle, V. K. Malik, A. J. Drew, R. H. Liu, G. Wu, X. H. Chen, and C. Bernhard, Phys. Rev. Lett. 101, 097011 (2008).
[28] Z.-C. Wang, C.-Y. He, S.-Q. Wu, Z.-T. Tang, Y. Liu, A. Ablimit, C.-M. Feng, and G.-H. Cao, J. Am. Chem. Soc. 138, 7856 (2016).

[29] Z. Wang, C. He, Z. Tang, S. Wu, and G. Cao, Sci. China Mater. 60, 83 (2017).

[30] Z.-C. Wang, Y. Liu, S.-Q. Wu, Y.-T. Shao, Z. Ren, and G.-H. Cao, Phys. Rev. B 99, 144501 (2019).

[31] B. Xu, Z. C. Wang, E. Sheveleva, F. Lyzwa, P. Marsik, G. H. Cao, and C. Bernhard, Phys. Rev. B 99, 125119 (2019).

[32] A. Sihvola, Electromagnetic Mixing Formulas and Applications (Institution of Electrical Engineers, London, 1999).

[33] T. G. Mayerhöfer, Z. Shen, R. Keding, and J. L. Musfeldt, Phys. Rev. B 71, 184116 (2005).

[34] J. Schützmann, S. Tajima, S. Miyamoto, Y. Sato, and R. Hauff, Phys. Rev. B 52, 13665 (1995).

[35] C. C. Homes, T. Timusk, D. A. Bonn, R. Liang, and W. N. Hardy, Can. J. Phys. 73, 663 (1995).

[36] D. Munzar, T. Holden, and C. Bernhard, Phys. Rev. B 67, 020501(R) (2003).

[37] D. Wu, W. Hong, C. Dong, X. Wu, Q. Sui, J. Huang, Q. Gao, C. Li, C. Song, H. Luo, C. Yin, Y. Xu, X. Luo, Y. Cai, J. Jia, Q. Wang, Y. Huang, G. Liu, S. Zhang, F. Zhang, F. Yang, Z. Wang, Q. Peng, Z. Xu, X. Qiu, S. Li, H. Luo, J. Hu, L. Zhao, and X. J. Zhou, arXiv:2001.04082.

[38] B. P. P. Mallett, P. Marsik, D. Munzar, C. Bernhard, and A. Dubroka, Phys. Rev. B 99, 054513 (2019).

[39] S. V. Dordevic, E. J. Singley, J. H. Kim, M. B. Maple, S. Komiya, S. Ono, Y. Ando, T. Rõõm, R. Liang, D. A. Bonn, W. N. Hardy, J. P. Carbotte, C. C. Homes, M. Strongin, and D. N. Basov, Phys. Rev. B 69, 094511 (2004).

[40] J. Chaloupka, C. Bernhard, and D. Munzar, Phys. Rev. B 79, 184513 (2009).

[41] M. Rotter, M. Tegel, and D. Johrendt, Phys. Rev. Lett. 101, 107006 (2008).

[42] S. Avci, O. Chmaissem, D. Y. Chung, S. Rosenkranz, E. A. Goremychkin, J. P. Castellan, I. S. Todorov, J. A. Schlueter, H. Claus, A. Daoud-Aladine, D. D. Khalyavin, M. G. Kanatzidis, and R. Osborn, Phys. Rev. B 85, 184507 (2012).

[43] A. E. Böhmer, F. Hardy, L. Wang, T. Wolf, P. Schweiss, and C. Meingast, Nat. Commun. 6, 7911 (2015).

[44] N. Ni, M. E. Tillman, J.-Q. Yan, A. Kracher, S. T. Hannahs, S. L. Bud'ko, and P. C. Canfield, Phys. Rev. B 78, 214515 (2008).

[45] P. C. Canfield, S. L. Bud'ko, N. Ni, J. Q. Yan, and A. Kracher, Phys. Rev. B 80, 060501(R) (2009).

[46] N. Ni, A. Thaler, J. Q. Yan, A. Kracher, E. Colombier, S. L. Bud'ko, P. C. Canfield, and S. T. Hannahs, Phys. Rev. B 82, 024519 (2010).

[47] T. Goltz, V. Zinth, D. Johrendt, H. Rosner, G. Pascua, H. Luetkens, P. Materne, and H.-H. Klauss, Phys. Rev. B 89, 144511 (2014).

[48] W. R. Meier, Q.-p. Ding, A. Kreyssig, S. L. Bud'ko, A. Sapkota, K. Kothapalli, V. Borisov, R. Valentí, C. D. Batista, P. P. Orth, R. M. Fernandes, A. I. Goldman, Y. Furukawa, A. E. Böhmer, and P. C. Canfield, npj Quantum Mater. 3, 5 (2018).

[49] Q.-P. Ding, W. R. Meier, J. Cui, M. Xu, A. E. Böhmer, S. L. Bud'ko, P. C. Canfield, and Y. Furukawa, Phys. Rev. Lett. 121, 137204 (2018). 